\title{
Two-sided fractional quaternion Fourier transform and its application
}

\author{
Zunfeng $\mathrm{Li}^{1 *} \mathbb{D}$, Haipan Shi ${ }^{2}$ and Yuying Qiao ${ }^{2}$
}

"Correspondence:

zunfeng928@163.com

${ }^{1}$ College of Science, Hebei

University of Science and

Technology, Shijiazhuang, Hebei Province 050018, P.R. China

Full list of author information is available at the end of the article

\begin{abstract}
In this paper, we introduce the two-sided fractional quaternion Fourier transform (FrQFT) and give some properties of it. The main results of this paper are divided into three parts. Firstly we give a definition of the FrQFT. Secondly based on properties of the two-sided QFT, we study the relationship between the two-sided QFT and the two-sided FrQFT, and give some differential properties of the two-sided FrQFT and the Parseval identity. Finally, we give an example to illustrate the application of the two-sided FrQFT and its inverse transform in solving partial differential equations.
\end{abstract}

Keywords: Two-sided fractional quaternion Fourier transform; Differential properties; Parseval identity; Partial differential equations

\section{Introduction}

There are two aspects of the generalization of the classical Fourier transform: one is to the high dimensional space, the other is to the fractional Fourier transform. The quaternion Fourier transform (QFT) is one of the generalized forms of the classical Fourier transform in high dimensional space and has been proved to be very useful in signal processing, nonmarginal color image processing, electromagnetism, multi-channel processing, quantum mechanics, and partial differential systems. Many scholars have done a lot of research on the QFT and got many excellent results. In recent years, some properties of the QFT and the two-sided QFT have been studied [4-11].

In 2007, Hitzer [6] researched the QFT properties useful for applications to differential equations, image processing and optimized numerical implementations and studied the general linear transformation behavior of the QFT with matrices. In 2010, Hitzer [7] derived a directional uncertainty principle for quaternion-valued functions subject to the QFT. In 2016, Hitzer [4] defined the FT on the quaternion domain and analyzed its main properties, including quaternion dilation, modulation, shift properties and Parseval identities. In 2017, Haoui and Fahlaoui [2] presented the Heisenberg inequality and Hardy's theorem for the two-sided QFT. In [11], Yang et al. studied uncertainty principles of the QFT under the polar coordinate form. In [1], Bahri proposed the uncertainty principle for the two-sided QFT. That uncertainty principle described that the spread of a quaternionvalued function and its two-sided QFT was inversely proportional. sharing, adaptation, distribution and reproduction in any medium or format, as long as you give appropriate credit to the original author(s) and the source, provide a link to the Creative Commons licence, and indicate if changes were made. The images or other third party material in this article are included in the article's Creative Commons licence, unless indicated otherwise in a credit line to the material. If material is not included in the article's Creative Commons licence and your intended use is not permitted by statutory regulation or exceeds the permitted use, you will need to obtain permission directly from the copyright holder. To view a copy of this licence, visit http://creativecommons.org/licenses/by/4.0/. 
On the basis of the above work, we give some properties of the two-sided FrQFT and its application. The structure of the article is as follows: in the second section, we introduce the basic knowledge related to the quaternion Fourier analysis. In the third section, we first give a definition of the two-sided FrQFT. Then based on the nature of the two-sided QFT, we study the relationship between the two-sided QFT and the two-sided FrQFT. We give some differential properties, shift properties of the two-sided FrQFT and Parseval identity. Finally, we give an example to illustrate the application of the two-sided FrQFT and its inverse transform in solving partial differential equations.

\section{Preliminaries}

Let $\mathbb{R}^{2}$ be a real linear space with basis $\left\{e_{1}, e_{2}\right\}$, the quaternion algebra $\mathbb{H}$ which is an associative and noncommutative algebra structure spanned by

$$
\left\{1, e_{1}, e_{2}, e_{1} e_{2}\right\}
$$

And basis elements satisfy the following multiplication laws:

$$
\left\{\begin{array}{l}
e_{i}^{2}=-1, \quad i=1,2 ; \\
e_{1} e_{2}=-e_{2} e_{1}=e_{12} ; \\
e_{12}{ }^{2}=e_{12} e_{12}=-1 ; \\
e_{2} e_{12}=-e_{12} e_{2}=e_{1} ; \\
e_{12} e_{1}=-e_{1} e_{12}=e_{2} .
\end{array}\right.
$$

Every quaternion

$$
q=q_{0}+q_{1} e_{1}+q_{2} e_{2}+q_{3} e_{12} \in \mathbb{H}, \quad q_{0}, q_{1}, q_{2}, q_{3} \in \mathbb{R},
$$

has a quaternion conjugate $\bar{q}=q_{0}-q_{1} e_{1}-q_{2} e_{2}-q_{3} e_{12}$, where $(q)_{0}=q_{0}$.

For arbitrary $p, q \in \mathbb{H}, \overline{p q}=\overline{q p}$.

For quaternion-valued functions $f, g: \mathbb{R}^{2} \rightarrow \mathbb{H}$, the quaternion-valued inner product is defined by

$$
(f, g)=\int_{\mathbb{R}^{2}} f(\mathbf{x}) \overline{g(\mathbf{x})} d \mathbf{x}
$$

and the real scalar part is

$$
\langle f, g\rangle=\frac{1}{2}[(f, g)+(g, f)]=\int_{\mathbb{R}^{2}}(f(\mathbf{x}) g(\mathbf{x}))_{0} d \mathbf{x},
$$

where $d \mathbf{x}=d x_{1} d x_{2}$.

In particular, when $f=g$, this leads to

$$
\|f\|_{L^{2}\left(\mathbb{R}^{2} ; \mathbb{H}\right)}^{2}=(f, f)=\langle f, f\rangle=\int_{\mathbb{R}^{2}}|f(\mathbf{x})|^{2} d \mathbf{x} .
$$


Definition 2.1 ([12]) For any infinitely differentiable function $f(x)$, if

$$
\int_{-\infty}^{\infty} \delta(x) f(x) d x=\lim _{\varepsilon \rightarrow 0^{+}} \int_{-\infty}^{\infty} \delta_{\varepsilon}(x) f(x) d x
$$

we call the weak limit of $\delta_{\varepsilon}(x)$ a $\delta$ function and denote $\lim _{\varepsilon \rightarrow 0^{+}} \delta_{\varepsilon}(x)=\delta(x)$, where

$$
\delta_{\varepsilon}(x)= \begin{cases}0, & x<0 \\ \frac{1}{\varepsilon}, & 0 \leq x \leq \varepsilon \\ 0, & x>\varepsilon .\end{cases}
$$

Here are some properties of $\delta$ function as described below.

Lemma 2.1 ([12]) Suppose that $\lim _{\varepsilon \rightarrow 0+} \delta_{\varepsilon}(x)=\delta(x)$, then

(1) $\int_{-\infty}^{\infty} \delta(x) d x=1$;

(2) $\frac{1}{2 \pi} \int_{-\infty}^{\infty} e^{j w x} d w=\delta(x), \frac{1}{2 \pi} \int_{-\infty}^{\infty} e^{j w\left(x-x_{0}\right)} d w=\delta\left(x-x_{0}\right)$;

(3) $\int_{-\infty}^{\infty} \delta(x) f(x) d x=f(0), \int_{-\infty}^{\infty} \delta\left(x-x_{0}\right) f(x) d x=f\left(x_{0}\right)$.

Definition 2.2 We define

$$
\begin{aligned}
L^{1}\left(\mathbb{R}^{2} ; \mathbb{H}\right) & =\left\{f: \mathbb{R}^{2} \rightarrow \mathbb{H}\left|\int_{\mathbb{R}^{2}}\right| f(\mathbf{x}) \mid d \mathbf{x}<\infty\right\}, \\
L^{2}\left(\mathbb{R}^{2} ; \mathbb{H}\right) & =\left\{f:\left.\mathbb{R}^{2} \rightarrow \mathbb{H}\left|\int_{\mathbb{R}^{2}}\right| f(\mathbf{x})\right|^{2} d \mathbf{x}<\infty\right\} .
\end{aligned}
$$

Next, we define the space

$$
S\left(\mathbb{R}^{2} ; \mathbb{H}\right)=\left\{f \in C^{\infty}\left(\mathbb{R}^{2} ; \mathbb{H}\right)\left|\sup _{x \in \mathbb{R}^{2}}\left(1+|x|^{k}\right)\right| \partial^{\alpha} f(x) \mid<\infty\right\},
$$

where $C^{\infty}\left(\mathbb{R}^{2} ; \mathbb{H}\right)$ is the set of all infinitely differentiable functions from $\mathbb{R}^{2}$ to $\mathbb{H}$, and $\alpha=$ $\left(\alpha_{1}, \alpha_{2}\right), \alpha_{1}, \alpha_{2}, k \in \mathbb{Z}_{+}$.

For convenience, we divide $f \in \mathbb{H}$ into two parts as follows.

Lemma 2.2 Let $e_{l} \in \mathbb{H}, l=1$, 2. For any $f \in \mathbb{H}$, we define

$$
f_{+e_{l}}=\frac{1}{2}\left(f+e_{l} f e_{l}\right), \quad f_{-e_{l}}=\frac{1}{2}\left(f-e_{l} f e_{l}\right) .
$$

Then $f=f_{+e_{l}}+f_{-e_{l}}$, and $\overline{f_{+e_{l}}}=\bar{f}_{+e_{l}}, \overline{f_{-e_{l}}}=\bar{f}_{-e_{l}}$.

Lemma 2.3 ([3]) Let $\alpha \in \mathbb{R}$ and $e_{l} \in \mathbb{H}$ with $e_{l}^{2}=-1$. We have a natural generalization of Euler's formula in quaternion analysis as follows:

$$
e^{e_{l}^{\alpha}}=\cos \alpha+e_{l} \sin \alpha .
$$

Theorem 2.1 Suppose $e_{l}$ is as stated above, for any $f \in \mathbb{H}$, the following equation is always true 
(1) $e^{c e_{l}} f_{+e_{l}}=f_{+e_{l}} e^{-c e_{l}}, e^{c e_{l}} f_{-e_{l}}=f_{-e_{l}} e^{c e_{l}}$.

(2) $\sqrt{\frac{1-e_{l} \cot \theta_{i}}{2 \pi}} f_{+e_{l}}=f_{+e_{l} l} \sqrt{\frac{1+e_{l} \cot \theta_{i}}{2 \pi}}, \sqrt{\frac{1-e_{l} \cot \theta_{i}}{2 \pi}} f_{-e_{l}}=f_{-e_{l}} \sqrt{\frac{1-e_{l} \cot \theta_{i}}{2 \pi}}$, where $c \in \mathbb{R}, i=1,2$.

Proof (1) According to Lemma 2.2, we have

$$
\begin{aligned}
e^{c e_{l}} f_{+e_{l}} & =\left(\cos c+e_{l} \sin c\right) \frac{1}{2}\left(f+e_{l} f e_{l}\right) \\
& =\frac{1}{2}\left(f+e_{l} f e_{l}\right) \cos c+\frac{1}{2} e_{l}\left(f+e_{l} f e_{l}\right) \sin c \\
& =\frac{1}{2}\left(f+e_{l} f e_{l}\right) \cos c+\frac{1}{2}\left(e_{l} f e_{l}\left(-e_{l}\right)-f e_{l}\right) \sin c \\
& =\frac{1}{2}\left(f+e_{l} f e_{l}\right) \cos c+\frac{1}{2}\left(e_{l} f e_{l}+f\right)\left(-e_{l}\right) \sin c \\
& =\frac{f+e_{l} f e_{l}}{2}\left(\cos c-e_{l} \sin c\right)=f_{+e_{l}} e^{-c e_{l}} .
\end{aligned}
$$

Similarly, we have $e^{c e_{l}} f_{-e_{l}}=f_{-e_{l}} e^{c e_{l}}$.

(2) According to Lemma 2.2, we have

$$
\begin{aligned}
\sqrt{\frac{1-e_{l} \cot \theta_{i}}{2 \pi}} & =\sqrt{\frac{\sin \theta_{i}-e_{l} \cos \theta_{i}}{2 \pi \sin \theta_{i}}}=\sqrt{\frac{-e_{l}\left(e_{l} \sin \theta_{i}+\cos \theta_{i}\right)}{2 \pi \sin \theta_{i}}} \\
& =\sqrt{\frac{-e_{l} e^{e_{l} \theta_{i}}}{2 \pi \sin \theta_{i}}}=\sqrt{\frac{e^{-\frac{\pi}{2} e_{l}} e^{e_{l} \theta_{i}}}{2 \pi \sin \theta_{i}}}=\sqrt{\frac{e^{\left(-\frac{\pi}{2}+\theta_{i}\right) e_{l}}}{2 \pi \sin \theta_{i}}} .
\end{aligned}
$$

The above equation as a function of $\theta_{i}$ is periodic with $\pi$.

When $\theta_{i} \in(0, \pi), \sqrt{\frac{e^{\left(-\frac{\pi}{2}+\theta_{i}\right) e_{l}}}{2 \pi \sin \theta_{i}}}=\frac{e^{\frac{1}{2}\left(-\frac{\pi}{2}+\theta_{i}\right) e_{l}}}{\sqrt{2 \pi \sin \theta_{i}}}$.

According to Eq. (1), we have

$$
\frac{e^{\frac{1}{2}\left(-\frac{\pi}{2}+\theta_{i}\right) e_{l}}}{\sqrt{2 \pi \sin \theta_{i}}} f_{+e_{l}}=f_{+e_{l}} \frac{e^{\frac{1}{2}\left(\frac{\pi}{2}-\theta_{i}\right) e_{l}}}{\sqrt{2 \pi \sin \theta_{i}}}
$$

that is,

$$
\sqrt{\frac{1-e_{l} \cot \theta_{i}}{2 \pi}} f_{+e_{l}}=f_{+e_{l}} \sqrt{\frac{1+e_{l} \cot \theta_{i}}{2 \pi}} .
$$

When $\theta_{i} \in(-\pi, 0), \sqrt{\frac{e^{\left(-\frac{\pi}{2}+\theta_{i}\right) e_{l}}}{2 \pi \sin \theta_{i}}}=\frac{e^{\frac{1}{2}\left(\frac{\pi}{2}+\theta_{i}\right) e_{l}}}{\sqrt{-2 \pi \sin \theta_{i}}}$.

So we have $\sqrt{\frac{1-e_{l} \cot \theta_{i}}{2 \pi}} f_{+e_{l}}=f_{+e_{l}} \sqrt{\frac{1+e_{l} \cot \theta_{i}}{2 \pi}}$.

The other cases are similar.

Theorem 2.2 Let $e_{l} \in \mathbb{H}, l=1$, 2 . For any $f_{1}, f_{2} \in \mathbb{H}$, we have

$$
\begin{array}{ll}
e^{-\alpha e_{l}} f_{1+} \overline{f_{2+}}=f_{1+} \overline{f_{2+}} e^{-\alpha e_{l}}, & e^{-\alpha e_{l}} f_{1-} \overline{f_{2-}}=f_{1-} \overline{f_{2-}} e^{-\alpha e_{l}} . \\
e^{-\alpha e_{l}} f_{1-} \overline{f_{2+}}=f_{1-} \overline{f_{2+}} e^{\alpha e_{l}}, & e^{-\alpha e_{l}} f_{1_{1}} \overline{f_{2-}}=f_{1+} \overline{f_{2-}} e^{\alpha e_{l}} .
\end{array}
$$


Proof According to Lemma 2.2 and Theorem 2.1, we have

$$
\begin{aligned}
e^{-\alpha e_{l}} f_{1+} \overline{f_{2+}} & =f_{1+} e^{\alpha e_{l}} \overline{f_{2+}}=f_{1+} e^{\alpha e_{l}} \frac{1}{2}\left(\overline{f_{2}}+\overline{e_{l} f_{2} e_{l}}\right) \\
& =f_{1+} e^{\alpha e_{l}} \frac{1}{2}\left(\overline{f_{2}}+\overline{e_{l}} \overline{f_{2}} \overline{e_{l}}\right)=f_{1+} e^{\alpha e_{l}} \frac{1}{2}\left(\overline{f_{2}}+e_{l} \overline{f_{2}} e_{l}\right)=f_{1+} \overline{f_{2+}} e^{-\alpha e_{l}},
\end{aligned}
$$

where

$$
\begin{aligned}
e^{\alpha e_{l}} & \frac{1}{2}\left(\overline{f_{2}}+e_{l} \overline{f_{2}} e_{l}\right) \\
= & \left(\cos \alpha+e_{l} \sin \alpha\right) \frac{1}{2}\left(\overline{f_{2}}+e_{l} \overline{f_{2}} e_{l}\right) \\
= & \frac{1}{2}\left(\overline{f_{2}}+e_{l} \overline{f_{2}} e_{l}\right) \cos \alpha+\frac{1}{2}\left(e_{l} \overline{f_{2}}+e_{l}^{2} \overline{f_{2}} e_{l}\right) \sin \alpha \\
= & \frac{1}{2}\left(\overline{f_{2}}+e_{l} \overline{f_{2}} e_{l}\right) \cos \alpha+\frac{1}{2}\left(e_{l} \overline{f_{2}}\left(-e_{l}^{2}\right)-\overline{f_{2}} e_{l}\right) \sin \alpha \\
= & \frac{1}{2}\left(\overline{f_{2}}+e_{l} \overline{f_{2}} e_{l}\right) \cos \alpha+\frac{1}{2}\left(e_{l} \overline{f_{2}} e_{l}+\overline{f_{2}}\right)\left(-e_{l}\right) \sin \alpha \\
= & \frac{1}{2}\left(\overline{f_{2}}+e_{l} \overline{f_{2}} e_{l}\right)\left(\cos \alpha-e_{l} \sin \alpha\right)=\overline{f_{2+}} e^{-\alpha e_{l}} .
\end{aligned}
$$

Similarly, we have

$$
\begin{aligned}
& e^{-\alpha e_{l}} f_{1-} \overline{f_{2-}}=f_{1-} \overline{f_{2-}} e^{-\alpha e_{l}}, \\
& e^{-\alpha e_{l}} f_{1-} \overline{f_{2+}}=f_{1-} \overline{f_{2+}} e^{\alpha e_{l}}, \\
& e^{-\alpha e_{l}} f_{1+} \overline{f_{2-}}=f_{1+} \overline{f_{2-}} e^{\alpha e_{l}}
\end{aligned}
$$

\section{Some properties of the two-sided FrQFT}

In this section we state some properties of the two-sided FrQFT. We first give a definition of the two-sided FrQFT and its inverse transformation. Then we get the relationship between two-sided QFT and the two-sided FrQFT. Finally we study the properties of this transformation, such as the shift property, differential properties of functions and their image functions, and differential properties of kernel functions.

Definition 3.1 Suppose that the function $f \in L^{1}\left(\mathbb{R}^{2} ; \mathbb{H}\right)$. We define $p=\left(p_{1}, p_{2}\right)$-order twosided FrQFT as follows:

$$
\mathcal{F}_{\theta_{1}, \theta_{2}}\{f\}(\mathbf{w})=\int_{\mathbb{R}^{2}} K_{\theta_{1}}\left(x_{1}, w_{1}\right) f(\mathbf{x}) K_{\theta_{2}}\left(x_{2}, w_{2}\right) d \mathbf{x},
$$

where

$$
\begin{array}{ll}
K_{\theta_{1}}\left(x_{1}, w_{1}\right)=C_{\theta_{1}} e^{e_{1} \frac{x_{1}^{2}+w_{1}^{2}}{2} \cot \theta_{1}-e_{1} x_{1} w_{1} \csc \theta_{1},} & C_{\theta_{1}}=\sqrt{\frac{1-e_{1} \cot \theta_{1}}{2 \pi}}, \\
K_{\theta_{2}}\left(x_{2}, w_{2}\right)=C_{\theta_{2}} e^{e_{2} \frac{x_{2}^{2}+w_{2}^{2}}{2} \cot \theta_{2}-e_{2} x_{2} w_{2} \csc \theta_{2}}, & C_{\theta_{2}}=\sqrt{\frac{1-e_{2} \cot \theta_{2}}{2 \pi}},
\end{array}
$$

and $\theta_{i} \neq n \pi, p_{i}=\frac{2 \theta_{i}}{\pi}, i=1,2$. 
Definition 3.2 Suppose that $f \in L^{1}\left(\mathbb{R}^{2} ; \mathbb{H}\right)$. We define the inverse transformation of the two-sided FrQFT as follows:

$$
\mathcal{H}_{\theta_{1}, \theta_{2}}\{f\}(\mathbf{w})=\int_{\mathbb{R}^{2}} K_{-\theta_{1}}\left(x_{1}, w_{1}\right) f(\mathbf{x}) K_{-\theta_{2}}\left(x_{2}, w_{2}\right) d \mathbf{x},
$$

where

$$
\begin{array}{ll}
K_{-\theta_{1}}\left(x_{1}, w_{1}\right)=C_{-\theta_{1}} e^{-e_{1} \frac{x_{1}^{2}+w_{1}^{2}}{2} \cot \theta_{1}+e_{1} x_{1} w_{1} \csc \theta_{1}}, & C_{-\theta_{1}}=\sqrt{\frac{1+e_{1} \cot \theta_{1}}{2 \pi}}, \\
K_{-\theta_{2}}\left(x_{2}, w_{2}\right)=C_{-\theta_{2}} e^{-e_{2} \frac{x_{2}^{2}+w_{2}^{2}}{2} \cot \theta_{2}+e_{2} x_{2} w_{2} \csc \theta_{2}}, & C_{-\theta_{2}}=\sqrt{\frac{1+e_{2} \cot \theta_{2}}{2 \pi}},
\end{array}
$$

where $\theta_{i}(i=1,2)$ are as mentioned above.

When $p_{1}=p_{2}=1$, the two-sided FrQFT becomes the two-sided QFT.

We know that the two-sided QFT is defined as (see [1])

$$
\mathcal{F}\{f\}(\mathbf{w})=\int_{\mathbb{R}^{2}} e^{-e_{1} x_{1} w_{1}} f(\mathbf{x}) e^{-e_{2} x_{2} w_{2}} d \mathbf{x} .
$$

Its inverse transformation is defined as

$$
\mathcal{F}^{-1}\{f\}(\mathbf{x})=\frac{1}{(2 \pi)^{2}} \int_{\mathbb{R}^{2}} e^{e_{1} x_{1} w_{1}} f(\mathbf{w}) e^{e_{2} x_{2} w_{2}} d \mathbf{w}
$$

The Plancherel identity is given by

$$
\left(f_{1}, f_{2}\right)=\frac{1}{(2 \pi)^{2}}\left(\mathcal{F}\left\{f_{1}\right\}(\mathbf{w}), \mathcal{F}\left\{f_{2}\right\}(\mathbf{w})\right)
$$

Theorem 3.1 The two-sided FrQFT and the two-sided QFT have the following relationship:

$$
\begin{aligned}
\mathcal{F}_{\theta_{1}, \theta_{2}}\{f\}(\mathbf{w}) & \\
= & C_{\theta_{1}} e^{e_{1} \frac{w_{1}^{2}}{2} \cot \theta_{1}} \int_{\mathbb{R}^{2}} e^{e_{1} \frac{x_{1}^{2}}{2} \cot \theta_{1}-e_{1} x_{1} w_{1} \csc \theta_{1}} f(\mathbf{x}) e^{e_{2} \frac{x_{2}^{2}}{2} \cot \theta_{2}-e_{2} x_{2} w_{2} \csc \theta_{2}} d \mathbf{x} \\
& \times\left(C_{\theta_{2}} e^{e_{2} \frac{w_{2}^{2}}{2} \cot \theta_{2}}\right) \\
= & C_{\theta_{1}} e^{e_{1} \frac{w_{1}^{2}}{2} \cot \theta_{1}} \mathcal{F}\left\{e^{e_{1} \frac{x_{1}^{2}}{2} \cot \theta_{1}} f(\mathbf{x}) e^{e_{2} \frac{x_{2}^{2}}{2} \cot \theta_{2}}\right\}(\mathbf{w} \csc \theta)\left(C_{\theta_{2}} e^{e_{2} \frac{w_{2}^{2}}{2} \cot \theta_{2}}\right),
\end{aligned}
$$

where $\mathbf{w} \csc \theta=\left(w_{1} \csc \theta_{1}, w_{2} \csc \theta_{2}\right)$.

Theorem 3.2 Let $f, \mathcal{F}_{\theta_{1}, \theta_{2}}\{f\} \in L^{1}\left(\mathbb{R}^{2} ; \mathbb{H}\right)$ and $0<\left|\theta_{i}\right|<\pi, i=1$, 2. Then

$$
\left(\mathcal{H}_{\theta_{1}, \theta_{2}} \circ \mathcal{F}_{\theta_{1}, \theta_{2}}\right)\{f\}=\left(\mathcal{F}_{\theta_{1}, \theta_{2}} \circ \mathcal{H}_{\theta_{1}, \theta_{2}}\right)\{f\}=f .
$$


Proof According to the Fubini replacement theorem and Theorem 2.1, we have

$$
\begin{aligned}
& \left(\mathcal{H}_{\theta_{1}, \theta_{2}} \circ \mathcal{F}_{\theta_{1}, \theta_{2}}\right)\{f\}(\mathbf{y}) \\
& =\mathcal{H}_{\theta_{1}, \theta_{2}}\left\{\mathcal{F}_{\theta_{1}, \theta_{2}}\{f\}(w)\right\}(\mathbf{y}) \\
& =\int_{\mathbb{R}^{2}} K_{-\theta_{1}}\left(y_{1}, w_{1}\right)\left(\int_{\mathbb{R}^{2}} K_{\theta_{1}}\left(x_{1}, w_{1}\right) f(\mathbf{x}) K_{\theta_{2}}\left(x_{2}, w_{2}\right) d \mathbf{x}\right) K_{-\theta_{2}}\left(w_{2}, y_{2}\right) d \mathbf{w} \\
& =\int_{\mathbb{R}^{2}} \int_{\mathbb{R}^{2}} K_{-\theta_{1}}\left(y_{1}, w_{1}\right) K_{\theta_{1}}\left(x_{1}, w_{1}\right) f(\mathbf{x}) K_{\theta_{2}}\left(x_{2}, w_{2}\right) d \mathbf{x} K_{-\theta_{2}}\left(w_{2}, y_{2}\right) d \mathbf{w} \\
& =\int_{\mathbb{R}^{2}} \int_{\mathbb{R}^{2}} C_{\theta_{1}} C_{-\theta_{1}} e^{e_{1} \frac{x_{1}^{2}-y_{1}^{2}}{2} \cot \theta_{1}-e_{1}\left(x_{1}-y_{1}\right) w_{1} \csc \theta_{1}} f(\mathbf{x}) \\
& \times C_{\theta_{2}} C_{-\theta_{2}} e^{e_{2} \frac{x_{2}^{2}-y_{2}^{2}}{2} \cot \theta_{2}-e_{2}\left(x_{2}-y_{2}\right) w_{2} \csc \theta_{2}} d \mathbf{x} d \mathbf{w} \\
& =\left(\frac{1}{2 \pi}\right)^{2} \frac{1}{\left|\sin \theta_{1} \sin \theta_{2}\right|} \int_{\mathbb{R}^{2}} e^{e_{1} \frac{-y_{1}^{2}}{2} \cot \theta_{1}} e^{e_{1} y_{1} w_{1} \csc \theta_{1}} \int_{\mathbb{R}^{2}} e^{-e_{1} x_{1} w_{1} \csc \theta_{1}} e^{e_{1} \frac{x_{1}^{2}}{2} \cot \theta_{1}} \\
& \times f(\mathbf{x}) e^{-e_{2} x_{2} w_{2} \csc \theta_{2}} e^{e_{2} \frac{x_{2}^{2}}{2} \cot \theta_{2}} d \mathbf{x} e^{e_{2} \frac{-y_{2}^{2}}{2} \cot \theta_{2}} e^{e_{2} y_{2} w_{2} \csc \theta_{2}} d \mathbf{w} .
\end{aligned}
$$

Let $w_{i} \csc \theta_{i}=t_{i}$, then $w_{i}=\frac{t_{i}}{\sin \theta_{i}}, i=1,2$. According to (3.3) and (3.4), we have

$$
\begin{aligned}
& \left(\mathcal{H}_{\theta_{1}, \theta_{2}} \circ \mathcal{F}_{\theta_{1}, \theta_{2}}\right)\{f\}(\mathbf{y}) \\
& =e^{e_{1} \frac{y_{1}^{2}}{2} \cot \theta_{1}}\left(\frac{1}{2 \pi}\right)^{2} \int_{\mathbb{R}^{2}} e^{e_{1} y_{1} t_{1}} \int_{\mathbb{R}^{2}} e^{-e_{1} t_{1} x_{1}} e^{e_{1} \frac{x_{1}^{2}}{2} \cot \theta_{1}} f(\mathbf{x}) e^{e_{2} \frac{x_{2}^{2}}{2} \cot \theta_{2}} e^{-e_{2} t_{2} x_{2}} \\
& \quad \times d \mathbf{x} e^{e_{2} y_{2} t_{2}} d \mathbf{t} e^{e_{2} \frac{-y_{2}^{2}}{2} \cot \theta_{2}} \\
& =e^{e_{1} \frac{-y_{1}^{2}}{2} \cot \theta_{1}} \mathcal{F}^{-1}\left(\mathcal{F}\left\{e^{e_{1} \frac{y_{1}^{2}}{2} \cot \theta_{1}} f(\mathbf{y}) e^{e_{2} \frac{y_{2}^{2}}{2} \cot \theta_{2}}\right\}\right) e^{e_{2} \frac{-y_{2}^{2}}{2} \cot \theta_{2}} \\
& =f(\mathbf{y}) .
\end{aligned}
$$

Similarly we have $\left(\mathcal{F}_{\theta_{1}, \theta_{2}} \circ \mathcal{H}_{\theta_{1}, \theta_{2}}\right)\{f\}=f$. It means that $\mathcal{F}_{\theta_{1}, \theta_{2}}\{f\}(\mathbf{w})$ and $\mathcal{H}_{\theta_{1}, \theta_{2}}\{f\}(\mathbf{w})$ are inverse transformations of each other.

Next, we give some important properties of the two-sided FrQFT; we begin with the shift property.

Theorem 3.3 Let $f \in L^{1}\left(\mathbb{R}^{2} ; \mathbb{H}\right)$ and $\mathbf{t}=\left(t_{1}, t_{2}\right)$, the following property holds

$$
\begin{aligned}
& \mathcal{F}_{\theta_{1}, \theta_{2}}\{f(\mathbf{x}-\mathbf{t})\}(\mathbf{w}) \\
& =e^{e_{1} \frac{t_{1}^{2}}{2} \cot \theta_{1}-e_{1} t_{1} w_{1} \csc \theta_{1}} \mathcal{F}_{\theta_{1}, \theta_{2}}\left\{e^{e_{1} y_{1} t_{1} \cot \theta_{1}} f(\mathbf{y}) e^{e_{2} y_{2} t_{2} \cot \theta_{2}}\right\}(\mathbf{w}) \\
& \quad \times e^{e_{2} \frac{t_{2}^{2}}{2} \cot \theta_{2}-e_{2} t_{2} w_{2} \csc \theta_{2}} .
\end{aligned}
$$

Proof

$$
\begin{aligned}
\mathcal{F}_{\theta_{1}, \theta_{2}} & \{f(\mathbf{x}-\mathbf{t})\}(\mathbf{w}) \\
\quad= & \int_{\mathbb{R}^{2}} K_{\theta_{1}}\left(x_{1}, w_{1}\right) f(\mathbf{x}-\mathbf{t}) K_{\theta_{2}}\left(x_{2}, w_{2}\right) d \mathbf{x}
\end{aligned}
$$




$$
\begin{gathered}
=\int_{\mathbb{R}^{2}} C_{\theta_{1}} e^{e_{1} \frac{x_{1}^{2}+w_{1}^{2}}{2} \cot \theta_{1}-e_{1} x_{1} w_{1} \csc \theta_{1}} f(\mathbf{x}-\mathbf{t}) \\
\times C_{\theta_{2}} e^{e_{2} \frac{x_{2}^{2}+w_{2}^{2}}{2} \cot \theta_{2}-e_{2} x_{2} w_{2} \csc \theta_{2}} d \mathbf{x} .
\end{gathered}
$$

Let $x_{i}-t_{i}=y_{i}, i=1,2$, we have

$$
\begin{aligned}
\mathcal{F}_{\theta_{1}, \theta_{2}} & \{f(\mathbf{x}-\mathbf{t})\}(\mathbf{w}) \\
= & \int_{\mathbb{R}^{2}} C_{\theta_{1}} e^{e_{1} \frac{\left(y_{1}+t_{1}\right)^{2}+w_{1}^{2}}{2} \cot \theta_{1}-e_{1}\left(y_{1}+t_{1}\right) w_{1} \csc \theta_{1}} f(\mathbf{y}) \\
& \times C_{\theta_{2}} e^{e_{2} \frac{\left(y_{2}+t_{2}\right)^{2}+w_{2}^{2}}{2} \cot \theta_{2}-e_{2}\left(y_{2}+t_{2}\right) w_{2} \csc \theta_{2}} d \mathbf{y} \\
= & e^{e_{1} \frac{t_{1}^{2}}{2} \cot \theta_{1}-e_{1} t_{1} w_{1} \csc \theta_{1}} \int_{\mathbb{R}^{2}} C_{\theta_{1}} e^{e_{1} \frac{y_{1}^{2}+w_{1}^{2}}{2} \cot \theta_{1}-e_{1} y_{1} w_{1} \csc \theta_{1}} e^{e_{1} y_{1} t_{1} \cot \theta_{1}} f(\mathbf{y}) \\
& \times C_{\theta_{2}} e^{e_{2} \frac{y_{2}^{2}+w_{2}^{2}}{2} \cot \theta_{2}-e_{2} y_{2} w_{2} \csc \theta_{2}} e^{e_{2} y_{2} t_{2} \cot \theta_{2}} d \mathbf{y} e^{e_{2} \frac{t_{2}^{2}}{2} \cot \theta_{2}-e_{2} t_{2} w_{2} \csc \theta_{2}} \\
= & e^{e_{1} \frac{t_{1}^{2}}{2} \cot \theta_{1}-e_{1} t_{1} w_{1} \csc \theta_{1}} \mathcal{F}_{\theta_{1}, \theta_{2}}\left\{e^{e_{1} y_{1} t_{1} \cot \theta_{1}} f(\mathbf{y}) e^{e_{2} y_{2} t_{2} \cot \theta_{2}}\right\}(\mathbf{w}) \\
& \times e^{e_{2} \frac{t_{2}^{2}}{2} \cot \theta_{2}-e_{2} t_{2} w_{2} \csc \theta_{2}}
\end{aligned}
$$

In the following theorem we give the differential properties of the two-sided FrQFT. These conclusions are similar in nature to those of the classical FT, although they have different forms.

Theorem 3.4 Let $f \in S\left(\mathbb{R}^{2} ; \mathbb{H}\right)$, then, for each component $x_{s}$ and $w_{s}$ with $s=1,2$, the following relation is fulfilled:

$$
\begin{aligned}
& \mathcal{F}_{\theta_{1}, \ldots, \theta_{n}}\left\{\frac{\partial f}{\partial x_{s}}\right\}(\mathbf{w}) \\
& \quad= \begin{cases}-e_{1} \cot \theta_{1} \mathcal{F}_{\theta_{1}, \theta_{2}}\left\{x_{1} f\right\}(\mathbf{w})+e_{1} w_{1} \csc \theta_{1} \mathcal{F}_{\theta_{1}, \theta_{2}}\{f\}(\mathbf{w}), & s=1 ; \\
-\cot \theta_{2} \mathcal{F}_{\theta_{1}, \theta_{2}}\left\{x_{2} f\right\}(\mathbf{w}) e_{2}+w_{2} \csc \theta_{2} \mathcal{F}_{\theta_{1}, \theta_{2}}\{f\}(\mathbf{w}) e_{2}, & s=2 .\end{cases}
\end{aligned}
$$

Proof When $s=1$, using integration by parts we have

$$
\begin{aligned}
\mathcal{F}_{\theta_{1}, \theta_{2}} & \left\{\frac{\partial f}{\partial x_{1}}\right\}(\mathbf{w}) \\
= & \int_{\mathbb{R}^{2}} C_{\theta_{1}} e^{e_{1} \frac{x_{1}^{2}+w_{1}^{2}}{2}} \cot \theta_{1}-e_{1} x_{1} w_{1} \csc \theta_{1} \frac{\partial f(\mathbf{x})}{\partial x_{1}} C_{\theta_{2}} e^{e_{2} \frac{x_{2}^{2}+w_{2}^{2}}{2} \cot \theta_{2}-e_{2} x_{2} w_{2} \csc \theta_{2}} d \mathbf{x} \\
= & \int_{\mathbb{R}} \int_{\mathbb{R}}\left(C_{\theta_{1}} e^{e_{1} \frac{x_{1}^{2}+w_{1}^{2}}{2} \cot \theta_{1}-e_{1} x_{1} w_{1} \csc \theta_{1}} \frac{\partial f(\mathbf{x})}{\partial x_{1}} d x_{1}\right) C_{\theta_{2}} \\
& \times e^{e_{2} \frac{x_{2}^{2}+w_{2}^{2}}{2} \cot \theta_{2}-e_{2} x_{2} w_{2} \csc \theta_{2}} d x_{2} \\
= & \int_{\mathbb{R}}\left(\left.C_{\theta_{1}} e^{e_{1} \frac{x_{1}^{2}+w_{1}^{2}}{2} \cot \theta_{1}-e_{1} x_{1} w_{1} \csc \theta_{1}} f(\mathbf{x})\right|_{x_{1}=-\infty} ^{x_{1}=+\infty}\right. \\
& \left.-\int_{\mathbb{R}} \frac{\partial}{\partial x_{1}}\left(C_{\theta_{i}} e^{e_{1} \frac{x_{1}^{2}+w_{1}^{2}}{2} \cot \theta_{1}-e_{1} x_{1} w_{1} \csc \theta_{1}}\right) f(\mathbf{x}) d x_{1}\right)
\end{aligned}
$$




$$
\begin{aligned}
& \times e^{e_{2} \frac{x_{2}^{2}+w_{2}^{2}}{2} \cot \theta_{2}-e_{2} x_{2} w_{2} \csc \theta_{2}} d x_{2} \\
= & -\int_{\mathbb{R}^{2}} \frac{\partial}{\partial x_{1}}\left(C_{\theta_{1}} e^{e_{1} \frac{x_{1}^{2}+w_{1}^{2}}{2} \cot \theta_{1}-e_{1} x_{1} w_{1} \csc \theta_{1}}\right) f(\mathbf{x}) \\
& \times e^{e_{2} \frac{x_{2}^{2}+w_{2}^{2}}{2} \cot \theta_{2}-e_{2} x_{2} w_{2} \csc \theta_{2}} d \mathbf{x} \\
= & -e_{1} \cot \theta_{1} \mathcal{F}_{\theta_{1}, \theta_{2}}\left\{x_{1} f\right\}(\mathbf{w})+e_{1} w_{1} \csc \theta_{1} \mathcal{F}_{\theta_{1}, \theta_{2}}\{f\}(\mathbf{w}) .
\end{aligned}
$$

Using a similar calculation, the conclusion is valid when $s=2$.

Theorem 3.4 describes the relationship between the two-sided FrQFT of the derivative of a function $f$ and the two-sided FrQFT of the function $f$ itself.

Theorem 3.5 Suppose that $f \in S\left(\mathbb{R}^{2} ; \mathbb{H}\right)$. Then we have

$$
\frac{\partial \mathcal{F}_{\theta_{1}, \theta_{2}}\{f\}(\mathbf{w})}{\partial w_{s}}= \begin{cases}e_{1}\left[w_{s} \cot \theta_{s} \mathcal{F}_{\theta_{1}, \theta_{2}}\{f\}(\mathbf{w})-\csc \theta_{s} \mathcal{F}_{\theta_{1}, \theta_{2}}\left\{x_{s} f\right\}(\mathbf{w})\right], & s=1 \\ {\left[w_{s} \cot \theta_{s} \mathcal{F}_{\theta_{1}, \theta_{2}}\{f\}(\mathbf{w})-\csc \theta_{s} \mathcal{F}_{\theta_{1}, \theta_{2}}\left\{x_{s} f\right\}(\mathbf{w})\right] e_{2},} & s=2\end{cases}
$$

or

$$
\mathcal{F}_{\theta_{1}, \theta_{2}}\left\{x_{s} f(\mathbf{x})\right\}(\mathbf{w})= \begin{cases}\left(-e_{1} \frac{\partial}{\partial w_{1}} \sin \theta_{1}+w_{1} \cos \theta_{1}\right) \mathcal{F}_{\theta_{1}, \theta_{2}}\{f(\mathbf{x})\}(\mathbf{w}), & s=1 \\ \left(-\frac{\partial}{\partial w_{2}} \sin \theta_{2} e_{2}+w_{s} \cos \theta_{2}\right) \mathcal{F}_{\theta_{1}, \theta_{2}}\{f(\mathbf{x})\}(\mathbf{w}), & s=2 .\end{cases}
$$

Proof The proof is similar to that of Theorem 3.4, so the proof is not given here.

Theorem 3.5 describes the relationship between the derivative of the two-sided FrQFT of a function $f$ and the two-sided FrQFT of the function $f$ itself.

Some important differential properties of kernel functions $K_{\theta_{1}}\left(x_{1}, w_{1}\right)$ and $K_{\theta_{2}}\left(x_{2}, w_{2}\right)$ are stated in the following theorems.

Theorem 3.6 Suppose that $f \in S\left(\mathbb{R}^{2} ; \mathbb{H}\right)$,

$$
\begin{aligned}
& \triangle_{x_{1}}=\frac{\partial}{\partial x_{1}}-e_{1} x_{1} \cot \theta_{1}, \\
& \triangle_{x_{2}}=\frac{\partial}{\partial x_{2}}-x_{2} \cot \theta_{2} e_{2} .
\end{aligned}
$$

Then

$$
\begin{aligned}
& \triangle_{x_{1}}^{m} K_{\theta_{1}}\left(x_{1}, w_{1}\right)=e_{1}^{m}\left(-w_{1} \csc \theta_{1}\right)^{m} K_{\theta_{1}}\left(x_{1}, w_{1}\right), \\
& \triangle_{x_{2}}^{m} K_{\theta_{2}}\left(x_{2}, w_{2}\right)=\left(-w_{2} \csc \theta_{2}\right)^{m} K_{\theta_{2}}\left(x_{2}, w_{2}\right) e_{2}^{m} .
\end{aligned}
$$

Proof By simple calculation, we have

$$
\begin{aligned}
& \triangle_{x_{1}} K_{\theta_{1}}\left(x_{1}, w_{1}\right) \\
& \quad=\left(e_{1} x_{1} \cot \theta_{1}-e_{1} w_{1} \csc \theta_{1}-e_{1} x_{1} \cot \theta_{1}\right) K_{\theta_{1}}\left(x_{1}, w_{1}\right) \\
& \quad=-e_{1} w_{1} \csc \theta_{1} K_{\theta_{1}}\left(x_{1}, w_{1}\right) .
\end{aligned}
$$


Then, by induction, assuming

$$
\triangle_{x_{1}}^{m-1} K_{\theta_{1}}\left(x_{1}, w_{1}\right)=\left(-e_{1} w_{1} \csc \theta_{1}\right)^{m-1} K_{\theta_{1}}\left(x_{1}, w_{1}\right)
$$

is true, then

$$
\begin{aligned}
& \triangle_{x_{1}}^{m} K_{\theta_{1}}\left(x_{1}, w_{1}\right) \\
& \quad=\triangle_{x_{1}}\left(\triangle_{x_{1}}^{m-1} K_{\theta_{1}}\left(x_{1}, w_{1}\right)\right) \\
& =\triangle_{x_{1}}\left(\left(-e_{1} w_{1} \csc \theta_{1}\right)^{m-1} K_{\theta_{1}}\left(x_{1}, w_{1}\right)\right) \\
& =\left(-e_{1} w_{1} \csc \theta_{1}\right)^{m-1} \triangle_{x_{1}} K_{\theta_{1}}\left(x_{1}, w_{1}\right) \\
& =\left(-e_{1} w_{1} \csc \theta_{1}\right)^{m} K_{\theta_{1}}\left(x_{1}, w_{1}\right) \\
& =e_{1}^{m}\left(-w_{1} \csc \theta_{1}\right)^{m} K_{\theta_{1}}\left(x_{1}, w_{1}\right) .
\end{aligned}
$$

Using a similar calculation, we can prove that the other equation is valid.

Theorem 3.7 Let $f \in L^{1}\left(\mathbb{R}^{2} ; \mathbb{H}\right)$, for any $m_{1}, m_{2} \in \mathbb{Z}_{+}$, we have

$$
\begin{aligned}
& \triangle_{x_{1}}^{m_{1}} \triangle_{x_{2}}^{m_{2}}\left(K_{\theta_{1}}\left(x_{1}, w_{1}\right) K_{\theta_{2}}\left(x_{2}, w_{2}\right)\right) \\
& \quad=\left(-w_{1} \csc \theta_{1}\right)^{m_{1}} e_{1}^{m_{1}} K_{\theta_{1}}\left(x_{1}, w_{1}\right) K_{\theta_{2}}\left(x_{2}, w_{2}\right)\left(-w_{2} \csc \theta_{2}\right)^{m_{2}} e_{2}^{m_{2}} .
\end{aligned}
$$

Theorem 3.8 Let $f \in L^{1}\left(\mathbb{R}^{2} ; \mathbb{H}\right)$,

$$
\begin{aligned}
& \bar{\triangle}_{x_{1}}=\frac{\partial}{\partial x_{1}}+e_{1} x_{1} \cot \theta_{1}, \\
& \bar{\triangle}_{x_{2}}=\frac{\partial}{\partial x_{2}}+x_{2} \cot \theta_{2} e_{2} .
\end{aligned}
$$

Then

$$
\begin{aligned}
& \mathcal{F}_{\theta_{1}, \theta_{2}}\left\{\bar{\triangle}_{x_{1}}^{m} f\right\}(\mathbf{w})=e_{1}^{m}\left(w_{1} \csc \theta_{1}\right)^{m} \mathcal{F}_{\theta_{1}, \theta_{1}}\{f\}(\mathbf{w}), \\
& \mathcal{F}_{\theta_{1}, \theta_{2}}\left\{\bar{\triangle}_{x_{2}}^{m} f\right\}(\mathbf{w})=\left(w_{2} \csc \theta_{2}\right)^{m} \mathcal{F}_{\theta_{1}, \theta_{2}}\{f\}(\mathbf{w}) e_{2}^{m} .
\end{aligned}
$$

Proof Using integration by parts we get

$$
\begin{aligned}
\mathcal{F}_{\theta_{1}, \theta_{2}} & \left\{\bar{\triangle}_{x_{1}} f(\mathbf{x})\right\}(\mathbf{w}) \\
= & \int_{\mathbb{R}^{2}} K_{\theta_{1}}\left(x_{1}, w_{1}\right) \frac{\partial f(\mathbf{x})}{\partial x_{1}} K_{\theta_{2}}\left(x_{2}, w_{2}\right) d \mathbf{x} \\
& +\int_{\mathbb{R}^{2}} e_{1} x_{1} \cot \theta_{1} K_{\theta_{1}}\left(x_{1}, w_{1}\right) f(\mathbf{x}) K_{\theta_{2}}\left(x_{2}, w_{2}\right) d \mathbf{x} \\
= & \int_{\mathbb{R}}\left(\left.K_{\theta_{1}}\left(x_{1}, w_{1}\right) f(\mathbf{x})\right|_{x_{1} \rightarrow-\infty} ^{x_{1} \rightarrow \infty}-\frac{\partial K_{\theta_{1}}\left(x_{1}, w_{1}\right)}{\partial x_{1}} f(\mathbf{x}) d x_{1}\right) K_{\theta_{2}}\left(x_{2}, w_{2}\right) d x_{2} \\
& +e_{1} x_{1} \cot \theta_{1} \mathcal{F}_{\theta_{1}, \theta_{2}}\{f\}(\mathbf{w})
\end{aligned}
$$




$$
\begin{aligned}
& =\left(e_{1} w_{1} \csc \theta_{1}-e_{1} x_{1} \cot \theta_{1}\right) \mathcal{F}_{\theta_{1}, \theta_{2}}\{f\}(\mathbf{w})+e_{1} x_{1} \cot \theta_{1} \mathcal{F}_{\theta_{1}, \theta_{2}}\{f\}(\mathbf{w}) \\
& =e_{1} w_{1} \csc \theta_{1} \mathcal{F}_{\theta_{1}, \theta_{2}}\{f\}(\mathbf{w}) .
\end{aligned}
$$

Then, by induction, assuming

$$
\mathcal{F}_{\theta_{1}, \theta_{2}}\left\{\bar{\triangle}_{x_{1}}^{(m-1)} f\right\}(\mathbf{w})=\left(e_{1} w_{1} \csc \theta_{1}\right)^{m-1} \mathcal{F}_{\theta_{1}, \theta_{2}}\{f\}(\mathbf{w})
$$

is true, then

$$
\begin{aligned}
\mathcal{F}_{\theta_{1}, \theta_{2}}\left\{\bar{\triangle}_{x_{1}}^{m} f\right\}(\mathbf{w}) & =\mathcal{F}_{\theta_{1}, \theta_{2}}\left\{\bar{\triangle}_{x_{1}}^{(m-1)}\left(\bar{\triangle}_{x_{1}}\right) f\right\}(\mathbf{w}) \\
& =\left(e_{1} w_{1} \csc \theta_{1}\right)^{m-1} \mathcal{F}_{\theta_{1}, \theta_{2}}\left\{\bar{\triangle}_{x_{1}} f\right\}(\mathbf{w}) \\
& =\left(e_{1} w_{1} \csc \theta_{1}\right)^{m} \mathcal{F}_{\theta_{1}, \theta_{2}}\{f\}(\mathbf{w}) .
\end{aligned}
$$

Using a similar calculation, we can prove that the other equation is valid.

Lemma 3.1 Suppose that $f \in S\left(\mathbb{R}^{2} ; \mathbb{H}\right), \bar{\triangle}_{x}^{m_{1}, m_{2}}=\bar{\triangle}_{x_{1}}^{m_{1}} \bar{\triangle}_{x_{2}}^{m_{2}}$. Then, for any positive integers $m_{1}, m_{2}$, we have

$$
\mathcal{F}_{\theta_{1}, \theta_{2}}\left\{\bar{\triangle}_{x}^{m_{1}, m_{2}} f\right\}(\mathbf{w})=\left(w_{1} \csc \theta_{1}\right)^{m_{1}}\left(w_{2} \csc \theta_{2}\right)^{m_{2}} e_{1}^{m_{1}} \mathcal{F}_{\theta_{1}, \theta_{2}}\{f\}(\mathbf{w}) e_{2}^{m_{2}} .
$$

Lemma 3.1 describes the relationship between the two-sided FrQFT of the derivative of a function $f$ and the two-sided FrQFT of the function itself.

Lemma 3.2 describes the relationship between the derivative of the two-sided FrQFT of a function $f$ and the two-sided FrQFT of $\left(-e_{1} x_{1} \csc \theta_{1}\right)^{m_{1}} f$ and $\left(-x_{1} \csc \theta_{1}\right)^{m_{2}} f e_{2}^{m_{2}}$.

Lemma 3.2 Suppose that $f \in S\left(\mathbb{R}^{2} ; \mathbb{H}\right)$,

$$
\begin{aligned}
& \triangle_{w_{1}}=\frac{\partial}{\partial w_{1}}-e_{1} w_{1} \cot \theta_{1}, \\
& \triangle_{w_{2}}=\frac{\partial}{\partial w_{2}}-w_{2} \cot \theta_{2} e_{2} .
\end{aligned}
$$

Then, for any positive integers $m_{1}, m_{2}$, we have

$$
\Delta_{w_{i}} \mathcal{F}_{\theta_{1}, \theta_{2}}\{f\}(\mathbf{w})= \begin{cases}\mathcal{F}_{\theta_{1}, \theta_{2}}\left\{\left(-e_{1} x_{1} \csc \theta_{1}\right)^{m_{1}} f\right\}(\mathbf{w}), & i=1 \\ \mathcal{F}_{\theta_{1}, \theta_{2}}\left\{\left(-x_{2} \csc \theta_{2}\right)^{m_{2}} f e_{2}^{m_{2}}\right\}(\mathbf{w}), & i=2 .\end{cases}
$$

Lemma 3.3 Suppose that $f \in S\left(\mathbb{R}^{2} ; \mathbb{H}\right)$ and $\triangle_{w}^{m_{1}, m_{2}}=\triangle_{w_{1}}^{m_{1}} \triangle_{w_{2}}^{m_{2}}$. Then, for any positive integers $m_{1}, m_{2}$, we have

$$
\triangle_{w}^{m_{1}, m_{2}} \mathcal{F}_{\theta_{1}, \theta_{2}}\{f\}(\mathbf{w})=\mathcal{F}_{\theta_{1}, \theta_{2}}\left\{\left(-x_{1} \csc \theta_{1}\right)^{m_{1}}\left(-x_{2} \csc \theta_{2}\right)^{m_{2}} e_{1}^{m_{1}} f e_{2}^{m_{2}}\right\}(\mathbf{w}) .
$$

We will give the properties of inner and scalar products.

Theorem 3.9 Suppose that $f_{1}, f_{2} \in L^{2}\left(\mathbb{R}^{2} ; \mathbb{H}\right), f_{1+} \overline{f_{2-}}+f_{1} \overline{f_{2+}}=0$. Then we have

$$
\left(\mathcal{F}_{\theta_{1}, \theta_{2}}\left\{f_{1}\right\}, \mathcal{F}_{\theta_{1}, \theta_{2}}\left\{f_{2}\right\}\right)=\left(f_{1}, f_{2}\right)
$$


Proof By the definition of the inner product and the definition of the two-sided FrQFT, we get

$$
\begin{aligned}
& \left(\mathcal{F}_{\theta_{1}, \theta_{2}}\left\{f_{1}\right\}(\mathbf{w}), \mathcal{F}_{\theta_{1}, \theta_{2}}\left\{f_{2}\right\}(\mathbf{w})\right) \\
& =\int_{\mathbb{R}^{2}}\left(\mathcal{F}_{\theta_{1}, \theta_{2}}\left\{f_{1}\right\}(\mathbf{w}) \overline{\mathcal{F}_{\theta_{1}, \theta_{2}}\left\{f_{2}\right\}(\mathbf{w})}\right) d \mathbf{w} \\
& =\int_{\mathbb{R}^{2}}\left(\int_{\mathbb{R}^{2}} K_{\theta_{1}}\left(x_{1}, w_{1}\right) f(\mathbf{x}) K_{\theta_{2}}\left(x_{2}, w_{2}\right) d \mathbf{x}\right. \\
& \times \overline{\left.\int_{\mathbb{R}^{2}} K_{\theta_{1}}\left(x_{1}, w_{1}\right) f(\mathbf{y}) K_{\theta_{2}}\left(x_{2}, w_{2}\right) d \mathbf{y}\right)} d \mathbf{w} \\
& =\int_{\mathbb{R}^{2}}\left(\int_{\mathbb{R}^{2}} C_{\theta_{1}} e^{e_{1} \frac{x_{1}^{2}+w_{1}^{2}}{2} \cot \theta_{1}-e_{1} x_{1} w_{1} \csc \theta_{1}} f_{1}(\mathbf{x}) e^{e_{2} \frac{x_{2}^{2}+w_{2}^{2}}{2} \cot \theta_{2}} C_{\theta_{2}} e^{e_{2} \frac{w_{2}^{2}}{2} \cot \theta_{2}} d \mathbf{x}\right. \\
& \times \overline{\int_{\mathbb{R}^{2}} C_{\theta_{1}} e^{e_{1} \frac{x_{1}^{2}+w_{1}^{2}}{2}} \cot \theta_{1}-e_{1} x_{1} w_{1} \csc \theta_{1}} f_{1}(\mathbf{x}) e^{\left.e_{2} \frac{x_{2}^{2}+w_{2}^{2}}{2} \cot \theta_{2} C_{\theta_{2}} e^{e_{2} \frac{w_{2}^{2}}{2} \cot \theta_{2}} d \mathbf{y}\right) d \mathbf{w}} \\
& =\int_{\mathbb{R}^{2}} \int_{\mathbb{R}^{2}} \int_{\mathbb{R}^{2}} C_{\theta_{1}} e^{e_{1} \frac{x_{1}^{2}+w_{1}^{2}}{2} \cot \theta_{1}-e_{1} x_{1} w_{1} \csc \theta_{1}} f_{1}(\mathbf{x}) \\
& \times C_{\theta_{2}} C_{-\theta_{2}} e^{e_{2} \frac{x_{2}^{2}+w_{2}^{2}}{2} \cot \theta_{2}-e_{2} x_{2} w_{2} \csc \theta_{2}} e^{-e_{2} \frac{y_{2}^{2}+w_{2}^{2}}{2} \cot \theta_{2}+e_{2} y_{2} w_{2} \csc \theta_{2}} \\
& \times \overline{f_{2}(\mathbf{y})} C_{-\theta_{1}} e^{-e_{1} \frac{y_{1}^{2}+w_{1}^{2}}{2} \cot \theta_{1}+e_{1} y_{1} w_{1} \csc \theta_{1}} d \mathbf{x} d \mathbf{y} d \mathbf{w}
\end{aligned}
$$

note that $C_{\theta_{2}} C_{-\theta_{2}}=\frac{\csc \theta_{2}}{2 \pi}$,

$$
\int_{\mathbb{R}} C_{\theta_{2}} C_{-\theta_{2}} e^{e_{2}\left(y_{2}-x_{2}\right) w_{2} \csc \theta_{2}} d w_{2}=\delta\left(y_{2}-x_{2}\right),
$$

then using the properties of $\delta$, we have

$$
\int_{\mathbb{R}} C_{\theta_{2}} C_{-\theta_{2}} \delta\left(y_{2}-x_{2}\right) e^{e_{2} \frac{x_{2}^{2}-y_{2}^{2}}{2}} \overline{f_{2}(\mathbf{y})} d y_{2}=\overline{f_{2}\left(y_{1}, x_{2}\right)} .
$$

So

$$
\begin{aligned}
& \left(\mathcal{F}_{\theta_{1}, \theta_{2}}\left\{f_{1}\right\}(\mathbf{w}), \mathcal{F}_{\theta_{1}, \theta_{2}}\left\{f_{2}\right\}(\mathbf{w})\right) \\
& =\int_{\mathbb{R}^{2}} \int_{\mathbb{R}} \int_{\mathbb{R}} C_{\theta_{1}} e^{e_{1} \frac{x_{1}^{2}+w_{1}^{2}}{2} \cot \theta_{1}-e_{1} x_{1} w_{1} \csc \theta_{1}} f_{1}(\mathbf{x}) \overline{f_{2}\left(y_{1}, x_{2}\right)} \\
& \times C_{-\theta_{1}} e^{-e_{1} \frac{y_{1}^{2}+w_{1}^{2}}{2} \cot \theta_{1}+e_{1} y_{1} w_{1} \csc \theta_{1}} d \mathbf{x} d y_{1} d w_{1} .
\end{aligned}
$$

Now, let us write $f_{1} \overline{f_{2}}=f_{1+} \overline{f_{2+}}+f_{1-} \overline{f_{2-}}+f_{1+} \overline{f_{2-}}+f_{1-} \overline{f_{2+}}$.

Using the definition and properties of the function $\delta$ and the well-known conditions $f_{1+} \overline{f_{2-}}+f_{1-} \overline{f_{2+}}=0$, then, by Theorem 2.2, we get

$$
\begin{aligned}
\left(\mathcal{F}_{\theta_{1}, \theta_{2}} f_{1}(\mathbf{w}), \mathcal{F}_{\theta_{1}, \theta_{2}}\left\{f_{2}\right\}(\mathbf{w})\right) & =\int_{\mathbb{R}^{2}} f_{1}(\mathbf{y}) \overline{f_{2}(\mathbf{y})} d \mathbf{y} \\
& =\left(f_{1}, f_{2}\right) .
\end{aligned}
$$


In particular, when $f_{1}=f_{2}=f$, by Theorem 3.9, we immediately arrive at the following conclusion. Of course, the following equality can also be proved to be true by the definition of the norm.

Theorem 3.10 (Parseval identity) Suppose that $f \in L^{2}\left(\mathbb{R}^{2} ; \mathbb{H}\right)$. Then we have

$$
\|f\|_{L^{2}\left(R^{2} ; \mathbb{H}\right)}^{2}=\left\|\mathcal{F}_{\theta_{1}, \theta_{2}}\{f\}\right\|_{L^{2}\left(R^{2} ; \mathbb{H}\right)}^{2}
$$

Proof Using the definition of the norm

$$
\begin{aligned}
& \left\|\mathcal{F}_{\theta_{1}, \theta_{2}}\{f\}\right\|_{L^{2}\left(R^{2} ; \mathbb{H}\right)}^{2} \\
& =\int_{\mathbb{R}^{2}}\left|K_{\theta_{1}}\left(x_{1}, w_{1}\right) f(\mathbf{x}) K_{\theta_{2}}\left(x_{2}, w_{2}\right)\right|^{2} d \mathbf{x} \\
& =\int_{\mathbb{R}^{2}}|f(\mathbf{x})|^{2} d \mathbf{x} \\
& =\|f\|_{L^{2}\left(R^{2} ; \mathbb{H}\right)}^{2} .
\end{aligned}
$$

From this conclusion, we can see that the two-sided FrQFT has norm-preserving properties.

\section{The application of the two-sided fractional QFT}

Next, we give an application of differential properties of the two-sided FrQFT in solving partial differential equations.

Example. Find solutions to the following partial differential equations.

$$
\left(\frac{\partial}{\partial x_{1}}+e_{1} x_{1} \cot \theta_{1}\right)^{4} y(\mathbf{x})\left(\frac{\partial}{\partial x_{2}}+e_{2} x_{2} \cot \theta_{2}\right)^{5}{\overline{e_{2}}}^{5}-y(\mathbf{x})=f(\mathbf{x})
$$

where $f(x)$ is a known quaternion-valued function and $y(x)$ is an unknown quaternionvalued function.

Solution. Using differential properties of the two-sided FrQFT, we take the two-sided FrQFT at both sides of differential equation (4.1). Then, by Theorem 3.4, we have

$$
\begin{aligned}
& \left(e_{1} w_{1} \csc \theta_{1}\right)^{4} \mathcal{F}_{\theta_{1}, \theta_{2}}\{y(\mathbf{x})\}(\mathbf{w})\left(e_{2} w_{2} \csc \theta_{2}\right)^{5}{\overline{e_{2}}}^{5}-\mathcal{F}_{\theta_{1}, \theta_{2}}\{y(\mathbf{x})\}(\mathbf{w}) \\
& \quad=\mathcal{F}_{\theta_{1}, \theta_{2}}\{f(\mathbf{x})\}(\mathbf{w}) .
\end{aligned}
$$

Then

$$
\left[\left(w_{1} \csc \theta_{1}\right)^{4}\left(w_{2} \csc \theta_{2}\right)^{5}-1\right] \mathcal{F}_{\theta_{1}, \theta_{2}}\{y(\mathbf{x})\}(\mathbf{w})=\mathcal{F}_{\theta_{1}, \theta_{2}}\{f(\mathbf{x})\}(\mathbf{w}) .
$$

That is,

$$
\mathcal{F}_{\theta_{1}, \theta_{2}}\{y(\mathbf{x})\}(\mathbf{w})=\frac{\mathcal{F}_{\theta_{1} \theta_{2}}\{f(\mathbf{x})\}(\mathbf{w})}{\left(w_{1} \csc \theta_{1}\right)^{4}\left(w_{2} \csc \theta_{2}\right)^{5}-1} .
$$


According to the Fourier inverse transform of the two-sided FrQFT, we can get

$$
\begin{aligned}
y(\mathbf{x}) & \\
& =\mathcal{F}_{\theta_{1}, \theta_{2}}^{-1}\left\{\frac{\mathcal{F}_{\theta_{1}, \theta_{2}}\{y(\mathbf{x})\}(\mathbf{w})}{\left(w_{1} \csc \theta_{1}\right)^{4}\left(w_{2} \csc \theta_{2}\right)^{5}-1}\right\} \\
& =\int_{\mathbb{R}^{2}} K_{-\theta_{1}}\left(x_{1}, w_{1}\right) \frac{\mathcal{F}_{\theta_{1}, \theta_{2}}\{y(\mathbf{x})\}(\mathbf{w})}{\left(w_{1} \csc \theta_{1}\right)^{4}\left(w_{2} \csc \theta_{2}\right)^{5}-1} K_{-\theta_{2}}\left(x_{2}, w_{2}\right) d \mathbf{x} \\
& =\int_{\mathbb{R}^{2}} A(\mathbf{w}) \mathcal{F}_{\theta_{1}, \theta_{2}}\{y(\mathbf{x})\}(\mathbf{w}) K_{-\theta_{2}}\left(x_{2}, w_{2}\right) d \mathbf{x},
\end{aligned}
$$

where $A(\mathbf{w})=\frac{K_{-\theta_{1}}\left(x_{1}, w_{1}\right)}{\left(w_{1} \csc \theta_{1}\right)^{4}\left(w_{2} \csc \theta_{2}\right)^{5}-1}$.

\section{Conclusion}

Using the basic concepts of quaternion algebra we introduced a two-sided FrQFT. Important properties of the two-sided FrQFT such as shift, differential properties, Parseval identities were demonstrated.

But so far there are still some problems to be studied. Firstly, we mention the relationship between the integral expression of the two-sided $\operatorname{FrQFT}$ of $f$ when $\theta_{i}=n \pi$ and that when $\theta_{i} \neq n \pi$. Secondly, we mention that applications of the two-sided FrQFT in signal processing, non-marginal color image processing and electromagnetism etc. are not given.

\section{Acknowledgements}

We thank the editors and all reviewers for taking time out of their busy schedules to read our papers and thank them for valuable suggestions.

\section{Funding}

This work was supported by the Doctoral Foundation of Heibei University of Science and Technology (No. 1181348), the National Natural Science Foundation of China (No. 11871191), the Innovative Foundation Project of Hebei Province (No. CXZZBS2021066), the Natural Science Foundation of Hebei Province (No. A2020205008), and the Key Foundation of Hebei Normal University (No. L2018Z01).

Availability of data and materials

Not applicable.

\section{Competing interests}

The authors declare that they have no competing interests.

\section{Authors' contributions}

ZFL has presented the main purpose of the article. All authors read and approved the final manuscript.

\section{Author details}

${ }^{1}$ College of Science, Hebei University of Science and Technology, Shijiazhuang, Hebei Province 050018, P.R. China. ${ }^{2}$ College of Mathematics and Information Science, Hebei Normal University, Shijiazhuang, Hebei Province 050024, P.R. China.

\section{Publisher's Note}

Springer Nature remains neutral with regard to jurisdictional claims in published maps and institutional affiliations.

Received: 8 November 2020 Accepted: 26 April 2021 Published online: 14 July 2021

\section{References}

1. Bahri, M.: A modified uncertainty principle for two-sided quaternion Fourier transform. Adv. Appl. Clifford Algebras 26, 513-527 (2016)

2. El Haoui, Y., Fahlaoui, S.: The uncertainty principle for the two-sided quaternion Fourier transform. Mediterr. J. Math. $14,221-232(2017)$

3. El Haoui, Y., Fahlaoui, S.: Donoho-Stark's uncertainty principles in real Clifford algebras. Adv. Appl. Clifford Algebras 29, 94 (2019) 
4. Fei, M., Xu, Y., Yan, J.: Real Paley-Wiener theorem for the quaternion Fourier transform. Complex Var. Elliptic Equ. 62, 1072-1080 (2017)

5. Hitzer, E.: Quaternion Fourier transform on quaternion fields and generalizations. Adv. Appl. Clifford Algebras 17, 497-517 (2007)

6. Hitzer, E.: Directional uncertainty principle for quaternion Fourier transform. Adv. Appl. Clifford Algebras 20, 271-284 (2010)

7. Hitzer, E.: The quaternion domain Fourier transform and its properties. Adv. Appl. Clifford Algebras 26, 969-984 (2016)

8. Mustard, D.: Fractional convolution. J. Aust. Math. Soc. Ser. B 40, 257-265 (1998)

9. Sangwine, S.J.: Fourier transforms of colour images using quaternion, or hypercomplex numbers. Electron. Lett. 32, 1979-1980 (1996)

10. Sangwine, S.J.: Color image edge detector based on quaternion convolution. Electron. Lett. 34, 969-971 (1998)

11. Yang, Y., Dang, P., Qian, T.: Tighter uncertainty principles based on quaternion Fourier transform. Adv. Appl. Clifford Algebras 26, 479-497 (2016)

12. Zhang, Y.L.: Engineering Mathematics. Higher Education Press, Beijing (2012)

Submit your manuscript to a SpringerOpen ${ }^{\circ}$ journal and benefit from:

- Convenient online submission

- Rigorous peer review

- Open access: articles freely available online

- High visibility within the field

- Retaining the copyright to your article

Submit your next manuscript at $\gg$ springeropen.com 